\title{
Critically ill obstetric patients with hypertensive disorders of pregnancy: Room for improvement
}

Hypertensive disorders of pregnancy constitute a global problem. While they are reported to complicate $5-10 \%$ of pregnancies, it is considered that the incidence is rising, owing to a number of factors including the increasing occurrence of cardiac and metabolic diseases in the reproductive age group.$^{[1]}$ It is reported that 42000 women succumb to hypertensive disorders of pregnancy annually. ${ }^{[2]}$ The majority of these deaths occur in low- and middle-income countries, and it is rare to encounter deaths related to hypertensive disorders of pregnancy in wellresourced countries. High mortality rates largely relate to a lack of early recognition and/or timely administration of appropriate therapy for preeclampsia and eclampsia. In South Africa (SA), 18\% of maternal deaths are attributed to hypertensive disorders of pregnancy, and they comprise the most common direct cause of maternal deaths. ${ }^{[3,4]}$ The majority of these deaths have been assessed as preventable. ${ }^{[5]}$

The pathophysiology of pre-eclampsia has not yet been clearly explained. It is theorised that altered immune mechanisms and/or genetic predisposition lead to abnormal placentation, which triggers a cascade of interrelated processes that culminate in widespread endothelial dysfunction. The production of cytokines, increased thromboxane and a lack of prostacycline and nitric-oxide release are key drivers of this multisystem disease. A state of vasospasm, vasoconstriction, increased vascular reactivity, amplified coagulation function and a contracted intravascular fluid compartment can rapidly develop. As a result, there is a risk of reduced oxygen delivery and cellular hypoxia, which if unchecked can culminate in multi-organ dysfunction (renal, respiratory, neurological, cardiac, haematological, and hepatic) and uteroplacental insufficiency. Therefore, a subset of patients with pre-eclampsia and eclampsia need intensive care unit (ICU) admission.

There is general agreement that critically ill obstetric patients constitute an altogether unique population by virtue of the various anatomical, physiological and biochemical changes that occur in the gravid and puerperal state. ${ }^{[6-8]}$ This affects both management and patient outcomes. There is a further suggestion that the hypertensive subgroup of obstetric patients may itself exhibit unique characteristics. ${ }^{[9-11]}$

The management of pre-eclampsia has been comprehensively addressed in a recent SA guideline ${ }^{[5]}$ In essence, key considerations for severe pre-eclampsia include recognition of hypertension, appropriate management of acute severe hypertension, surveillance, judicious fluid management (dose and type of fluid), magnesium sulphate administration and appropriate timing of delivery.

In this issue, Gama et al. ${ }^{[12]}$ describe the outcomes in a retrospective cohort of patients with hypertensive disorders of pregnancy admitted to a tertiary ICU, and compare this with a previous report from the same unit. ${ }^{[11,12]}$ In the 4-year period reviewed, $44 \%(n=102)$ of obstetric admissions to the ICU had a hypertensive disorder of pregnancy. The vast majority of admissions were eclamptic (55/80). Overall mortalities of $11.6 \%$ and $11.1 \%$ were observed for overall hypertensive disorders of pregnancy and eclampsia, respectively. Failure to use magnesium sulphate, a lowest recorded Glasgow Coma Scale (GCS) score <10 and respiratory failure were found to be significantly associated with non-survival. Early onset of hypertension, a previous pregnancy, HIV infection, failure to use magnesium sulphate, a lowest recorded GCS score $<10$ and a need for anticonvulsants other than magnesium sulphate or benzodiazepines were found to be significantly associated with poor outcomes (death or GCS score $\leq 14$ on ICU discharge). The study highlights the fact that cases of hypertensive disorders of pregnancy comprise a substantive proportion of ICU admissions, and that the associated mortality remains unacceptably high.

Compared with previous reports from the same unit, it is evident that the disease landscape has changed over the last 3 decades. ${ }^{[11,13]}$ Platteau et al. ${ }^{[13]}$ initially reported that over a 1 -year period, hypertensive disorders of pregnancy comprised 69\% (55/80) of obstetric admissions, with all but 2 of these patients presenting with eclampsia. The mortality was $18 \%(10 / 55)$. A second report, conducted over a 42 -month period, demonstrated an eclampsia mortality of $10.5 \%(n=11 / 105){ }^{[11]}$ In this second report, the GCS score and organ failure score were found to be good predictors of outcome, and the importance of the neurological management of these patients was highlighted. ${ }^{[11]}$

In Gama et al.s ${ }^{[12]}$ current report, the increase in the proportion of pre-eclampsia suggests that perhaps patients are presenting earlier (prior to the development of eclampsia). This may be the result of the introduction of a national policy of free public maternal care services. In developed countries where antenatal care is accessible and appropriately utilised, the hypertensive disease profile is different from that in developing countries. ${ }^{[6,14]}$ With adequate antenatal care provision, the vast majority of patients present with pre-eclampsia rather than eclampsia.

The identification of certain factors (previous pregnancy, time of onset of hypertension, HIV infection, respiratory failure) as associated with mortality or poor neurological outcomes by Gama et al. ${ }^{[12]}$ is interesting. These associations are hypothesis-generating, and warrant further exploration in a prospective larger cohort. Patients with hypertensive disorders of pregnancy are at risk of multisystem complications and poor outcome. There is a need to prospectively evaluate this population to ascertain risk factors, and identify appropriate interventions by a multidisciplinary team in these high-risk patients.

It is difficult to categorically conclude that the mortality due to hypertensive disorders of pregnancy has remained unchanged. In the last two decades in critical care, haemodynamic monitoring and management in many domains, including ventilation, sedation, glycaemic control and fluid management has evolved considerably. One would therefore expect mortality to have declined. However, this expected decline may have been confounded by various factors, including delays in access to ICU admission, or inappropriate therapy prior to ICU admission. Resource constraints and inefficiencies in referrals are also issues that warrant consideration. The authors' suggestion of a management protocol is highly relevant. Their ICU needs to reach out to its referral units so that patient management is optimised from the time of initial recognition of this disease, as devastating consequences may follow inappropriate management.

Well-resourced countries have demonstrated that it is possible to achieve good outcomes in patients with hypertensive disorders of pregnancy. From an SA perspective, the burden of hypertensive disorders of pregnancy needs to be addressed in a multi-pronged manner that includes access to healthcare (antenatal clinics, obstetric units and ICUs), early recognition of disease, education of healthcare 
providers, implementation of surveillance protocols, efficient referral services and appropriate management at all levels of healthcare.

F Paruk, MB ChB, Cert Crit Care (SA), FCOG (SA), PhD (Clin Med) Department of Critical Care, Faculty of Health Sciences, University of Pretoria, South Africa

Fathima.Paruk@up.ac.za

South Afr J Crit Care 2019;35(2):43-44. https://doi.org/10.7196/SAJCC.2019.v35i2.419

1. Hutcheon JA, Lisonkova S, Joseph KS. Epidemiology of pre-eclampsia and other hypertensive disorders of pregnancy. Best Pract Clin Obstetric Gynaecol 2011:25(4):391-403. https://doi. org/10.1016/j.bpobgyn.2011.01.006

2. Vousden N, Lawley E, Seed PT, et al. Incidence of eclampsia and related complications across 10 low- and middle-resource geographical regions: Secondary analysis of a cluster randomised controlled trial. PLoS Med 2019;16(3):e1002775. https://doi/10.1371/journal.pmed.1002775

3. Moodley J. Maternal deaths due to hypertensive disorders of pregnancy: Data from the 2014 2016 Saving Mothers Report. Obstetric Gynaecol Forum 2018;28(3):28-32.

4. National Department of Health, South Africa. Saving Mothers Report 2017. Pretoria: NDoH, 2017:5.
5. Moodley J, Soma-Pillay P, Buchmann E, et al. Hypertensive disorders of pregnancy: 2019 national guideline. S Afr Med J 2019;109(3 Suppl 1):S3-S16. https://doi.org/10.7196/SAMJ.2019. v109i3.14104

6. Mabie WC, Sibai BM. Treatment in an obstetric intensive care unit. Am J Obstet Gynecol 1990; 162(1):1-4. https://doi.org/10.1016/0002-9378(90)90808-k

7. Lapinsky SE, Kruczynski K, Seaward GR et al. Critical care management of the obstetric patient. Can J Anaesth 1997;44(3):325-329. https://doi.org/10.1007/BF03015374

8. Afessa B, Green B, Delke I, Koch K. Systemic inflammatory response syndrome, organ failure and outcome in critically ill obstetric patients treated in an ICU. Chest 2001:120(4):1271-1277. https:// doi.org/10.1378/chest.120.4.1271

9. Hazelgrove JF, Price C, Pappachan J, Smith GB. Multicentre study of obstetric admissions to 14 intensive care units in southern England. Crit Care Med 2001;29(4):770-775. https://doi. org/10.1097/00003246-200104000-00016

10. Zakalik G, Chena A, Marengo J, Fernandez R. Population of obstetric patients admitted in ICU. World Fed J Crit Care 2005;22(Suppl 1):82 [Abstract].

11. Bhagwanjee S, Paruk F, Moodley J, Muckart DJJ. Intensive care unit morbidity and mortalit from eclampsia: An evaluation of the Acute Physiology and Chronic Health Evaluation I score and the Glasgow Coma Scale Score. Crit Care Med 2000;28(1):120-124. https://doi. org/10.1097/00003246-200001000-00020

12. Gama S, Sebitloane M, De Vasconcellos. Outcomes of patients admitted to the intensive care unit for complications of hypertensive disorders of pregnancy at a South African tertiary hospital - a 4-year retrospective review. S Afr J Crit Care 2019;35(2):64-71. https://doi.org/10.7196 SAJCC.2019.v35i2.001

13. Platteau P, Engelhardt T, Moodley J, Muckart DJJ. Obstetric and gynaecological patient in an intensive care unit: A 1-year review. Trop Doct 1997;27(4):202-206. https://doi. org/10.1177/004947559702700406

14. Kilpatrick SJ, Matthay MA. Obstetric patients requiring critical care. Chest 1992:101(5):14071412. https://doi.org/10.1378/chest.101.5.1407 